\title{
AVALIAÇÃO DE TÉCNICAS FISIOTERAPÊUTICAS NO TRATAMENTO DO LINFEDEMA PÓS-CIRURGIA DE MAMA EM MULHERES
}

\author{
Meirelles MCCC ${ }^{1}$, Mamede MV ${ }^{2}$, Souza L ${ }^{3}$ e Panobianco MS ${ }^{2}$ \\ ${ }^{1}$ Curso de Fisioterapia, Universidade de Ribeirão Preto, Ribeirão Preto, SP - Brasil \\ ${ }^{2}$ Departamento de Enfermagem Materno-Infantil e Saúde Pública, Escola de Enfermagem de Ribeirão Preto, \\ Universidade de São Paulo - USP, Ribeirão Preto, SP - Brasil \\ ${ }^{3}$ Departamento de Medicina Social, Faculdade de Medicina de Ribeirão Preto, USP, Ribeirão Preto, SP - Brasil \\ Correspondência para: Prof ${ }^{\mathrm{a}} \mathrm{Dr}^{\mathrm{a}}$ Marislei Sanches Panobianco, Departamento de Enfermagem Materno-Infantil e Saúde \\ Pública, Escola de Enfermagem de Ribeirão Preto, USP, Av. Bandeirantes, 3900, CEP 14040-902, Ribeirão Preto, \\ SP - Brasil
}

Recebido: 08/08/2005 - Revisado: 26/06/2006 - Aceito: 14/09/2006

\begin{abstract}
RESUMO
Objetivo geral: Verificar, em um grupo de mulheres submetidas à cirurgia por câncer de mama, a efetividade do tratamento do linfedema, por um período de até dois anos. Objetivo específico: Avaliar o volume do braço tratado aos 6, 12, 18 e 24 meses após o tratamento do linfedema. Método: As participantes foram atendidas em um serviço de reabilitação especializado e submetidas ao tratamento do linfedema, com drenagem linfática manual, enfaixamento compressivo funcional, orientações de autocuidado, automassagem, uso da braçadeira elástica e exercício. Pelas medidas do volume dos membros, nos períodos determinados, 36 mulheres foram avaliadas após a fase intensiva, 22 após 6 meses, 15 após 12 meses, 6 após 18 meses e 11 aos 24 meses. A adesão às estratégias de autocuidado e exercícios foi avaliada através de um questionário. Testes estatísticos foram usados para buscar correlação entre redução do linfedema e alguns fatores como idade, escolaridade, grau do linfedema, tipo de cirurgia, entre outros. Resultados: Houve redução do linfedema e essa se manteve ao longo dos períodos estudados. Não houve correlação entre redução do linfedema e fatores como idade, estado civil, escolaridade, tipo de cirurgia, índice de massa corporal, grau do linfedema, radioterapia, circunferência, hipertensão arterial ou limitação articular e não houve adesão às estratégias de autocuidado com o braço, realização de exercícios, automassagem e uso de braçadeira elástica pela maioria das mulheres. Conclusões: É importante realizar novas pesquisas que analisem o papel de cada uma dessas orientações na evolução do linfedema após a fase intensiva do tratamento, de forma sistemática e controlada.
\end{abstract}

Palavras-chave: linfedema, neoplasias mamárias, reabilitação.

\section{ABSTRACT \\ Evaluation of Physiotherapeutic Techniques for Treating Lymphedema Following Breast Cancer Surgery in Women}

General objective: To verify the effectiveness of lymphedema treatment over a two-year period, in a group of women who underwent breast cancer surgery. Specific objective: To evaluate the volume of the treated arm at 6, 12, 18 and 24 months after the lymphedema treatment. Method: The participants were attended at a specialized rehabilitation service. They underwent lymphedema treatment consisting of manual lymphatic drainage and functional compressive bandaging and received guidance regarding self-care, self-massage, elastic sleeve use and exercise. They were evaluated by means of limb volume measurement at the following times: thirty-six women after the intensive phase, twenty-two after six months, fifteen after twelve months, six after eighteen months and eleven after twenty-four months. Adherence to the self-care strategies and exercises was evaluated by means of a questionnaire. Statistical tests were used to seek correlations between lymphedema reduction and some factors such as age, schooling, lymphedema grade, type of surgery, etc. Results: There was a reduction in lymphedema and this was maintained throughout the period analyzed. There was no correlation between lymphedema reduction and factors such as age, marital status, schooling, type of surgery, body mass index, lymphedema grade, radiotherapy, circumference, arterial hypertension or joint limitation. Moreover, most of the women did not adhere to the self-care strategies, arm exercises, self-massage and elastic sleeve use. Conclusion: It is important to conduct new studies, for systematic and controlled analysis of the role of this guidance in relation to lymphedema evolution following the intensive phase of treatment.

Key words: lymphedema, breast neoplasms, rehabilitation. 


\section{INTRODUÇÃO}

As mulheres submetidas à retirada cirúrgica de linfonodos axilares para o tratamento do câncer de mama estão sujeitas a complicações, entre elas, o linfedema de braço $^{1}$, definido como uma condição crônica, na qual existe acúmulo excessivo de líquido, com alta concentração proteica no interstício ${ }^{2}$ e de tratamento e reversibilidade difíceis e complexos.

Até o momento não existe um padrão universal para a mensuração do linfedema, e isso contribui para uma grande diversidade de resultados a respeito da sua incidência ${ }^{3}$. A incidência média, porém, inclusive no Brasil, fica em torno de $40 \%{ }^{4,5}$.

A alteração básica para a formação do linfedema devese à falência do sistema linfático. O aumento anormal da concentração de proteínas no interstício, resulta em um edema de alta concentração proteica ${ }^{6}$. A principal causa do linfedema pós-cirurgia mamária para o tratamento do câncer de mama é a retirada dos linfonodos axilares; alguns fatores como a radioterapia, as complicações pós-operatórias, a infecção, a linfangite, o nível da radicalidade da técnica cirúrgica e outros são relatados na literatura como fatores desencadeantes ou agravantes do linfedema, o que indica sua natureza multifatorial ${ }^{7,8,9}$.

Entre as complicações do linfedema pós-tratamento para o câncer de mama está a diminuição da capacidade de distensibilidade do tecido subcutâneo das estruturas envolvidas, como ombro, cotovelo, pulso e mão do lado comprometido, com prejuízo de movimentos e diminuição de amplitude. Isso pode causar desde um simples incômodo a uma forte dor no braço; a mulher pode ter suas atividades prejudicadas, a saúde cutânea e a subcutânea comprometidas e maior suscetibilidade a infecções no braço devido a ferimentos, picadas, ranhuras etc, em decorrência à diminuição da capacidade de regeneração do tecido ${ }^{10}$.

O linfedema interfere também na auto-imagem, no relacionamento marital e familiar, causa problemas de aceitabilidade social e pode levar a um linfangiossarcoma. Entre as possibilidades terapêuticas estão o tratamento conservador e a cirurgia, que somente é utilizada nos casos de maior gravidade. $\mathrm{O}$ tratamento conservador pode ser medicamentoso e fisioterapêutico. Quando o tratamento do linfedema é feito a tempo e adequadamente, diminui o potencial para complicações, além de facilitar a melhora $^{10,11,12,13,14,15}$.

O enfrentamento desse problema, na prática profissional, tem sido uma constante preocupação para quem trabalha com reabilitação de mulheres submetidas à cirurgia por câncer de mama, e a sua solução ainda está longe de ser alcançada.

Protocolos fisioterapêuticos para o tratamento do linfedema, que incluem drenagem linfática manual (DLM), enfaixamento compressivo funcional (ECF), exercícios, orientações ao autocuidado e à automassagem e uso de braçadeira elástica, divididos em uma fase intensiva e outra de manutenção do tratamento, mostram que a fase intensiva de tratamento é eficiente para reduzir significativamente o linfedema dessas mulheres, e que esta redução se dá, principalmente, na primeira semana de tratamento, sendo que após a terceira semana, a redução ocorre de maneira pouco significativa ${ }^{16,17}$.

Na fase de manutenção do tratamento, vários fatores podem interferir negativamente, tanto em relação à manutenção dos resultados obtidos, quanto à diminuição do linfedema, entre eles destaca-se o não cumprimento das orientações sobre o cuidado com o braço e a pele, a não aderência à realização dos exercícios e da automassagem e ao uso da braçadeira elástica ${ }^{16}$.

O objetivo geral deste estudo foi verificar, em um grupo de mulheres, a manutenção da efetividade do tratamento do linfedema pós-cirurgia por câncer de mama, com a utilização de DLM, ECF, orientações para o autocuidado, a automassagem, o uso de braçadeira elástica e a realização de exercícios, em um período de até dois anos. Os objetivos específicos foram: 1) verificar a redução do volume do braço no período de 6, 12, 18 e 24 meses após a fase intensiva de tratamento; 2) identificar fatores que possam estar relacionados à redução do volume do braço, tais como idade, estado civil, escolaridade, índice de massa corporal, grau do edema.

\section{METODOLOGIA}

Este trabalho foi aprovado pelo Comitê de Ética em Pesquisa da Escola de Enfermagem de Ribeirão Preto/USP e as participantes assinaram o termo de consentimento livre e esclarecido (of.CEP-EERP/USP - 151/2001).

Participaram do estudo 36 mulheres atendidas em um serviço especializado em reabilitação de mulheres com câncer de mama, as quais atendiam aos seguintes critérios: terem sido submetidas à cirurgia mamária e linfadenectomia axilar unilateral, apresentarem $3 \mathrm{~cm}$ ou mais de diferença entre as medidas dos braços à perimetria e terem completado a fase intensiva do tratamento do linfedema. Essas mulheres foram avaliadas aos 6, 12, 18 e 24 meses após o término dessa fase.

\section{Fases do tratamento}

Fase intensiva: esta fase constou de DLM, ECF, exercícios e automassagem e orientações sobre cuidados com o braço. A DLM e o ECF foram realizados pelo terapeuta, três vezes por semana, com o objetivo de descongestionar e criar novos caminhos entre os capilares linfáticos, aumentar a pressão e diminuir a filtração para o interstício, favorecendo a absorção do linfedema. Além disso, o enfaixamento evita o retorno do líquido deslocado pela DLM, devendo, porém, ser funcional para que a contração muscular seja possível, permitindo o efeito bomba da musculatura. As faixas são retiradas apenas na ocasião do novo enfaixamento. As mulheres foram orientadas a realizar em casa, até 2 vezes 
por dia, os exercícios e a automassagem com o objetivo de intensificar os efeitos obtidos pela DLM. Os exercícios visam à amplitude total do movimento, respeitando, porém, o limite individual. A automassagem consiste em massagear suavemente, em círculos, as duas regiões de linfonodos mais próximas, ou seja, a axila oposta ao linfedema e a virilha homolateral; seguir realizando manobras em semicírculos nas regiões compreendidas entre os grupos de linfonodos íntegros e a região edemaciada. As mulheres também recebem orientações quanto a estratégias de autocuidado com braço e mão e de como manter a pele hidratada e íntegra, visando evitar infecções e outras complicações que podem agravar o quadro e /ou diminuir a resposta ao tratamento.

Fase de manutenção: após a fase intensiva, os atendimentos passam a ser progressivamente reduzidos. As mulheres receberam orientações no sentido de manter os cuidados com o braço, os exercícios e a automassagem, a qual, nos primeiros dias dessa fase, passam a ser supervisionadas pelo terapeuta. Nessa fase, e foi recomendado que fizessem uso de uma braçadeira elástica, em substituição ao enfaixamento.

\section{Avaliação do linfedema}

O volume dos braços foi calculado a partir das medidas das circunferências de ambos os membros superiores, em sete pontos distribuídos ao longo do braço e antebraço. Essas medidas foram utilizadas para o cálculo do volume aproximado dos seis cones truncados, formados nos pontos de medidas das circunferências. A soma dessas seis partes dá o volume total do membro.

O volume foi calculado no início e no final da fase intensiva e próximo aos 6, 12, 18 ou 24 meses após a mesma, nos retornos das mulheres ao serviço. Nesses mesmos períodos, aplicou-se também um questionário para avaliar seu grau de aderência ao uso regular da braçadeira elástica, à realização dos exercícios e da automassagem, conforme elas haviam sido orientadas anteriormente.

Em cada ocasião calculou-se o volume do braço edemaciado com relação ao braço sem edema.

A redução do edema na fase final do tratamento intensivo foi definida por:

\section{Redução fi $=\frac{\text { aumento do volume no final }}{\text { aumento do volume no início }} \times \mathbf{1 0 0 \%}$}

A redução do edema na ocasião $t(t=6,12,18,24$ meses), em relação ao início do tratamento, foi definida por:

aumento do volume no início -

$$
\text { Redução ti }=\frac{\text { aumento do volume na ocasião } t}{\text { aumento do volume no início }} \times \mathbf{1 0 0 \%}
$$

Em relação ao final do tratamento, a redução foi definida por:

$$
\text { Redução fi }=\frac{\begin{array}{l}
\text { aumento do volume no início }- \\
\text { aumento do volume na ocasião } t
\end{array}}{\text { aumento do volume no final }} \times \mathbf{1 0 0 \%}
$$

Os dados como idade, estado civil, escolaridade, índice de massa corporal, grau do linfedema, radioterapia, diferença entre os braços, limitação articular, hipertensão arterial, obtidos a partir do prontuário da paciente, foram analisados para determinar se interfeririam na redução do aumento do volume do braço, ao longo dos períodos analisados. Os testes estatísticos utilizados para tal análise foram: o teste de correlação por postos de Spearman para o fator idade, o teste de Kruskal-Wallis para o fator tipo de cirurgia e o teste de Mann-Whitney para os demais fatores.

\section{RESULTADOS}

As 36 mulheres tinham de 32 a 80 anos, com idade média de 56,5 anos; 7 (19,4\%) mulheres foram submetidas à cirurgia de mama do tipo radical; 18 (50\%) do tipo radical modificada e 11 (30,6\%) do tipo conservadora; 6 (16,7\%) mulheres apresentavam linfedema na fase III, e as restantes (83,3\%), na fase II; 30 (83,3\%) realizaram radioterapia; 22 (61,1\%) eram obesas; 12 (33,3\%) apresentavam excesso de peso e 2 (5,6\%) tinham peso normal. A diferença média das circunferências entre os braços foi de 5,72 cm; 6 (16,7\%) apresentavam limitação de amplitude de movimento do braço homolateral à cirurgia; 16 (44,4\%) mulheres apresentavam hipertensão arterial sistêmica; 15 (41,7\%) residiam no município estudado, 19 (52,8\%) em cidades vizinhas e 2 (5,6\%) em outro Estado. Todas as 36 mulheres foram avaliadas ao final da fase intensiva do tratamento do linfedema, sendo que $22(61,1 \%)$ foram avaliadas 6 meses após esse período; 15 (41,7\%) aos 12 meses após; 6 (16,7\%) 18 meses após e 11 (30,7\%) 24 meses após. Apenas 4 (11,1\%) foram avaliadas nos 4 períodos.

\section{Redução do linfedema}

Fase intensiva: essa fase durou, em média, 4,41 semanas. A diferença de volume entre o braço sadio e o edemaciado, no início do tratamento, variou de 0,12 a 1,23 litros, com uma diferença média de 0,28 litros. Ao final da fase intensiva do tratamento, o grupo estudado apresentou uma redução média de 30,5\% entre os volumes dos braços sadio e edemaciado.

Redução do linfedema aos 6, 12, 18, e 24 meses: aos 6 meses, a redução média do linfedema, ao final da fase intensiva das 22 (61,1\%) mulheres avaliadas nesse período, foi de 30,5\%; 6 meses após, a redução média foi de 30,4\% em relação ao início do tratamento. Tomando por base o final da fase intensiva, 6 meses após, houve um aumento médio do linfedema de 5,7\%. 
Após 12 meses, foram avaliadas 15 (41,7\%) mulheres, sendo que a redução média do linfedema, ao final da fase intensiva, foi de 36,9\% para este grupo e, 12 meses após, a redução média foi de 19,8\%, em relação ao início do tratamento; havendo, portanto, um aumento médio do linfedema de 16,6\%.

Após 18 meses, foram avaliadas 6 (16,7\%) mulheres. A redução média do linfedema, ao final da fase intensiva, foi de 42,6\% e, 18 meses após, a redução média foi de 63,5\%, em relação ao início do tratamento. A redução média entre esses tempos de avaliação foi de 53,5\%.

Aos 24 meses, foram avaliadas 11 (30,6\%) mulheres. A redução média do linfedema, ao final da fase intensiva, foi de 38,4\% e, 24 meses após, a redução média foi de 24,0\%. Tomando por base o final da fase intensiva, houve um aumento médio do linfedema de 6,0\% (Tabela 1).

Tabela 1. Médias das reduções fi, ti e tf dos períodos estudados.

\begin{tabular}{ccccc}
\hline Tempo & n & Redução fi & Redução ti & Redução tf \\
\hline 6 meses & 22 & 30,5 & 30,4 & $-5,7$ \\
12 meses & 15 & 36,9 & 19,8 & $-16,6$ \\
18 meses & 06 & 42,6 & 63,5 & 53,5 \\
24 meses & 11 & 38,4 & 24,0 & $-6,0$ \\
\hline
\end{tabular}

Para atender ao objetivo deste trabalho, de verificar a manutenção da efetividade do tratamento do linfedema pós-cirurgia por câncer de mama, em um período de até dois anos, utilizou-se o teste de Wilcoxon, por meio do qual comparou-se a redução obtida em cada período estudado (6, 12, 18 e 24 meses) com os volumes antes do início do tratamento, após a fase intensiva e com zero.

A Tabela 2 apresenta a distribuição das medianas dos períodos estudados e os valores de p do teste de Wilcoxon, da Redução fi (no caso da redução ti) e da Redução tf comparada com zero.
Os altos valores de p dos testes da Redução ti versus a Redução fi, bem acima do nível de significância de 0,05, dão base para constatar que não há diferença significante entre a redução após o tempo de 6, 12, 18 e 24 meses e a redução após a fase intensiva de tratamento, indicando que o tratamento mantém sua eficiência no transcorrer dos 24 meses seguintes. Essa constatação é reforçada pela comparação das medianas das Reduções obtidas em cada período em relação ao início do tratamento (Redução ti) com a mediana do final da fase intensiva do tratamento (33,7 que é a Redução fi). A comparação da mediana das Reduções tf com zero mostra que a queda na redução é maior aos 24 meses, ou seja, $-11,4 \%$. (Tabela 2).

Finalmente, testou-se a redução do volume aos 24 meses em relação ao início (Redução 24i) contra zero, a fim de verificar se o volume aos 24 meses é menor que o volume da fase inicial do tratamento. O teste de Wilcoxon revela significância $(\mathrm{p}=0,03)$, ou seja, até 24 meses após a fase intensiva do tratamento, o volume não retorna ao valor inicial do linfedema.

Os fatores como a idade, o estado civil, a escolaridade, o índice de massa corporal (IMC), o grau do linfedema, a radioterapia, a diferença em centímetros (cm) entre os braços, a limitação articular e a hipertensão arterial não mostraram interferência na redução do volume do linfedema ao final da fase intensiva ou aos 6, 12 e 24 meses após. Essa análise não foi realizada no grupo avaliado aos 18 meses devido ao número reduzido de sujeitos (06).

O questionário utilizado para identificar o grau de aderência das mulheres às orientações recebidas a respeito dos cuidados com o braço após a fase intensiva do tratamento do linfedema foi aplicado em 5 mulheres, aos 6 meses da fase intensiva; em 5 mulheres, aos 12 meses; em 2 mulheres, aos 18 meses e em 8 mulheres, aos 24 meses. Das 20 (55,5\%) mulheres que responderam ao questionário, somente 4 (20\%) delas referiram realizar todas as atividades (realização de exercícios, automassagem e uso regular da braçadeira elástica). Outras 4 mulheres referiram não realizar nenhuma e as demais realizavam uma ou duas atividades.

Tabela 2. Medianas das Reduções ti, Redução tf e valores de p do teste de Wilcoxon dos períodos estudados.

\begin{tabular}{cccccc}
\hline Tempo & $\mathbf{n}$ & Redução ti & $\begin{array}{c}\text { Valor de p } \\
\text { (Reduçãoti X Redução fi) }\end{array}$ & Redução tf & $\begin{array}{c}\text { Valor de p } \\
\text { (Reduçãotf X zero) }\end{array}$ \\
\hline Final & 36 & 33,7 & & & \\
6 meses & 22 & 28,5 & 0,76 & $-2,2$ & 0,94 \\
12 meses & 15 & 24,9 & 0,53 & 4,7 & 0,73 \\
18 meses & 6 & 47,5 & 0,46 & 3,3 & 0,46 \\
24 meses & 11 & 20,6 & 0,72 & $-11,4$ & 0,93 \\
\hline
\end{tabular}


Em relação aos exercícios, 12 mulheres relataram realizálos. Aquelas que relataram não fazê-los, disseram que não tinham tempo, esqueciam-se ou achavam que eles não tinham o mesmo valor do que quando realizados no serviço de reabilitação.

Neste estudo, 10 (50\%) mulheres que responderam ao questionário faziam uso regular da braçadeira elástica e referiram que essa causava incômodos, desde o calor excessivo, até escoriações na axila, na região anterior do cotovelo, no polegar ou alergia na pele. Isso levou algumas mulheres a fazer uso intermitente da braçadeira elástica, ou mesmo abandonar a sua utilização. Entre as que faziam uso diário, a maioria não conseguia dormir com a braçadeira elástica.

No que diz respeito à automassagem, 9 (45\%) mulheres deste estudo a realizaram, e as que não a fizeram, referiram falta de tempo, incerteza quanto ao procedimento correto ou que não acreditavam poder alcançar resultados positivos com a automassagem.

\section{DISCUSSÃO}

Os resultados do tratamento do linfedema com técnicas fisioterapêuticas como drenagem linfática manual, enfaixamento compressivo funcional, vestimentas elásticas, exercícios e orientações de autocuidados e automassagem, utilizadas para o tratamento do linfedema revelam-se como bons, melhores e mais rápidos do que outros métodos não invasivos para o tratamento do linfedema. Contudo, os autores ${ }^{18}$ enfatizam que os bons resultados dependem do bom treinamento e cuidado do terapeuta e da colaboração do paciente após a fase intensiva de tratamento. Pesquisadores ${ }^{18}$ descrevem o resultado de uma série de casos constituídos de 16 braços linfedematosos tratados com técnicas semelhantes às usadas neste trabalho. Obtiveram uma redução média de 73\% na fase intensiva, depois de um ano, apresentaram uma redução de $80 \%$ sem nenhum outro tratamento e ainda descrevem, os mesmos autores, outra série de 56 pacientes que obtiveram uma redução média de $63 \%$ na fase intensiva e, 3 anos após, a redução foi de 64\%. Em outra série estudada pelos mesmos autores, a redução obtida com um mês de tratamento em 78 linfedemas unilateral de braços, foi de 64\%. A redução dependeu do grau do linfedema e da colaboração do paciente. Após um ano, 44 pacientes foram reavaliados e não apresentaram redução significativa nesse tempo ${ }^{18}$.

Apesar de não termos obtido valores tão altos de redução do linfedema ao longo do tempo, julgamos satisfatórios os resultados alcançados no presente estudo, uma vez que a aderência às estratégias de autocuidado por parte da clientela estudada não se deu de forma homogênea.

Para avaliar a eficiência do protocolo, a porcentagem de redução ao longo do período estudado em relação ao volume no início do tratamento (Redução ti) foi testada, em relação à redução obtida na fase intensiva do tratamento (redução fi). Dessa forma, se a diferença entre Redução ti e Redução fi não fosse estatisticamente significante ou fosse maior que zero, não haveria provas da ineficiência do protocolo. Uma vez que as Reduções tf de cada tempo estejam próximas de zero ou maior que zero, isso significa que o protocolo foi eficiente, já que a diferença entre os braços pouco variou após o tratamento ou até diminuiu.

Os fatores descritos na literatura como predisponentes ou complicantes do linfedema ${ }^{4,19,20}$, como a idade, o estado civil, a escolaridade, o índice de massa corporal (IMC), o grau do linfedema, a radioterapia, a diferença em centímetros $(\mathrm{cm})$ entre os braços, a limitação articular e a hipertensão arterial não mostraram interferência na redução do volume do linfedema neste estudo, contudo vale ressaltar que o número de sujeitos avaliados foi diminuindo à medida que o tempo de acompanhamento passava e que as ocorrências de alguns fatores ficaram bastante reduzidas, a exemplo dos seguintes: não realização de radioterapia, tipo de cirurgia e a maior diferença de circunferência entre os braços ser menor do que $5 \mathrm{~cm}$. Nesses casos, o poder do teste em detectar diferenças significantes ficou fragilizado.

Com relação à prática de exercícios, 40\% das mulheres entrevistadas disseram não realizá-los por motivos como falta de tempo e esquecimento, o que é confirmado por um estudo com mulheres submetidas à cirurgia por câncer de mama, que observou que $100 \%$ delas percebem a prática dos exercícios como boa para sua saúde e, como incentivo para tal, apontaram a melhora do corpo e da mente; conhecimento sobre sua importância; presença de um profissional e suporte dos familiares. Contudo, como importante barreira para a prática de exercícios, a falta de vontade apareceu em 96,7\% das mulheres, além de falta de condições emocionais, atividades sociais, falta de tempo, efeitos colaterais da quimioterapia e radioterapia e queixas físicas ${ }^{21}$. Apesar das barreiras identificadas, pesquisadores relatam a melhora da qualidade de vida dessas mulheres quando elas participam de programas regulares de atividade física ${ }^{22,23}$.

Em relação à aderência ao uso da braçadeira, os dados mostram que $50 \%$ das mulheres faziam uso regularmente, apesar do incômodo causado. A principal estratégia adotada pelas mulheres para superar esse incômodo foi o uso intermitente da braçadeira. A não resolução desses incômodos foi o que causou o abandono do uso por muitas delas. Entre as que faziam uso diário, a maioria não conseguiu permanecer com a braçadeira durante a noite enquanto dormia.

Estudos afirmam que o resultado do tratamento para o linfedema depende da colaboração da paciente, especialmente quanto ao uso de vestes compressivas; reforçam que essa colaboração está relacionada ao esclarecimento que a paciente deve receber quanto aos motivos do uso da braçadeira e da impotância do ajuste da mesma ao braço. Sugerem ainda que a braçadeira deva ser substituída a cada 2-6 meses para se 
manter a compressão adequada ${ }^{24}$. A substituição da braçadeira não foi controlada nas mulheres acompanhadas neste estudo.

O uso da braçadeira elástica nas pacientes com linfedema deve ser encorajado, contudo não há consenso se essas braçadeiras precisam ser feitas sob medidas ou podem ser compradas nos tamanhos padronizados, bem como sobre o tanto de pressão que deva exercer sobre o braço. A recomendação tem variado entre três classes de compressão: 20-30mmHg; 30-40mmHg, e 40-50mmHg ${ }^{25}$.

Estudando a relação entre o grau de redução do volume do braço, a longo prazo, com a adesão da paciente ao uso da braçadeira elástica, assim como a conservação da elasticidade da mesma e a realização de exercícios, pesquisadores observaram que, para as pacientes $100 \%$ colaborativas, a redução aumentou de $63 \%$ para $79 \%$, em 3 anos de estudos, e para as não colaborativas diminuiu de $63 \%$ para $43 \%$. Essas diferenças foram consideradas significantes ${ }^{26}$.

Com relação às estratégias de autocuidado, estudos revelaram que a freqüência do uso das técnicas que exigem assistência, como massagens e bandagens, teve um declínio após o tratamento intensivo de quatro semanas, quando comparado com a adesão à realização dos exercícios e ao uso da braçadeira elástica ${ }^{27}$, fato esse que confirma os dados deste estudo. Somente $45 \%$ das mulheres realizaram automassagem, enquanto os exercícios eram realizados por $60 \%$ e o uso da braçadeira era feito por 50\% delas.

Devido à grande variabilidade de resposta à aderência às orientações, não foi possível estabelecer relação entre o cumprimento das orientações e a redução do linfedema, mas autores que procuraram estudar a relação entre o grau de redução do volume do braço, a longo prazo, com a colaboração da paciente quanto ao uso da braçadeira, conservação da mesma e realização de exercícios, encontraram que, para os pacientes $100 \%$ colaborativas, a redução aumentou de $63 \%$ para $79 \%$, nos 3 anos estudados e para os não colaborativos diminuiu de $63 \%$ para $43 \%$. Essa diferença foi considerada por eles bastante significante ${ }^{20}$.

Em outro estudo foram acompanhadas 239 pacientes portadoras de linfedema, tratadas com técnicas fisioterapêuticas semelhantes às utilizadas neste estudo, mas com uma fase intensiva que variou de 3 a 8 dias. Observouse uma redução do linfedema de membro superior que variou de 44 a $62 \%$ e de membro inferior de 42 a $40 \%$. Observouse ainda uma redução de $38 \%$ a $56 \%$ no membro superior e de $41 \%$ a $27 \%$ em membros inferiores a longo prazo (38 a 52 dias) e julgou-se que os benefícios foram mantidos através de automassagem, exercícios, auto-enfaixamento e uso contínuo de vestimentas de compressão ${ }^{26}$.

De qualquer forma, a redução do linfedema evidenciada nestes estudos é animadora, principalmente por se manter durante um longo período.

Frente aos resultados deste estudo, e de outros ${ }^{20,26,28}$, julga-se necessária a implementação, no atendimento às mulheres com linfedema, de estratégias de encorajamento para que as mesmas tenham uma maior colaboração na manutenção, ou ainda, na melhora dos resultados obtidos com o tratamento intensivo, incluindo a repetição periódica da fase intensiva de tratamento.

\section{CONCLUSÕES}

Os resultados deste estudo permitem concluir que: houve redução do linfedema e essa se manteve ao longo dos períodos estudados; não houve influência na redução do linfedema dos fatores como idade, estado civil, escolaridade, tipo de cirurgia, índice de massa corporal, grau do linfedema, radioterapia, circunferência, hipertensão arterial ou limitação articular; não houve adesão total às estratégias de autocuidado com o braço, realização de exercícios, automassagem e ao uso da braçadeira elástica por todas as mulheres estudadas.

Torna-se evidente a importância da realização de novas pesquisas que analisem o papel de cada uma dessas orientações na evolução do linfedema, após a fase intensiva do tratamento, de forma sistemática e controlada.

\section{REFERÊNCIAS BIBLIOGRÁFICAS}

1. Mamede MV. Assistência à mulher com câncer de mama: um novo enfoque assistencial. [Tese]. Ribeirão Preto (SP): Universidade de São Paulo. Escola de Enfermagem, 1991.

2. Rockson SG. Lymphedema. Am J of Med. 2001; (110): 288-94.

3. Földi E, Földi M, Clodius L. The lymphedema chaos: a lancet. Ann. Plast. Surg. 1989; (22): 505-15.

4. Vogelfang D. Linfologia básica. São Paulo: Ícone; 1995.

5. Brorson H. Liposuction gives complete reduction of chronic large arm lymphedema after breast cancer. Acta Oncol. 2000; (39): 407-20.

6. Panobianco MS. Acompanhamento dos três primeiros meses pós tratamento cirúrgico do câncer de mama: estudo das complicações e intercorrências associadas ao edema de braço. [dissertação]. Ribeirão Preto (SP): Universidade de São Paulo. Escola de Enfermagem, 1998.

7. Erickson VS, Pearson ML, Ganz PA, Adams J, Kahn KL. Arm edema in breast cancer patients. J Natl Cancer Inst. 2001; (2): 96-111.

8. Smith AH, Russo A, Muraca MG, Del Turco MR, Cardona G. Prognostic factors for lymphedema after primary treatment of breast carcinoma. Cancer. 2001; (92): 783-7.

9. Földi E, Földi M, Weissleder H. Conservative treatment of lymphoedema of the limbs. Angiol J Vasc Dis. 1985; (36): 17180.

10. Brennan MJ, Pompolo RW, Garden FH. Focused review: postmastectomy lymphedema. Arch Phys Med Rehabil. 1996; (77): 77-80.

11. Hoe AL, Iven D, Rooyle GT, Taylor I. Incidence of arm swelling following axillary clearance for breast cancer. Br J Surg. 1992; (79): 261-2. 
12. Bertelli G, Venturini M, Forno G, Macchiavello F, Dini D. An analysis of prognostic factors in response to conservative treatment of postmastectomy lymphedema. Surg Gynecol Obstet. 1992; (175): 455-60.

13. Woods MB, Tobin M, Mortimer MD. The psychosocial morbidity of breast cancer patients with lymphoedema. Cancer Nurs. 1995; (18): 467-71.

14. Humble CA. Lymphedema: incidence, pathophysiology, management, and nursing care. Cont Educ. 1995; (22): 1503-09.

15. Morgan RG, Casley-Smith JR, Mason MR. Complex physical therapy for the lymphoedematous arm. J Hand Surg. 1992; (17B): 437 - 41.

16. Meirelles MCCC. Linfedema pós-cirurgia por câncer de mama: avaliação de um protocolo de tratamento [Dissertação]. Ribeirão Preto (SP): Universidade de São Paulo. Escola de Enfermagem de Ribeirão Preto, 1998.

17. McNeely ML, Magee DJ, Lees AW, Bagnall KM, Haykowsky $\mathrm{M}$, Hanson $\mathrm{J}$. The addition of manual lymph drainage to compression therapy for breast cancer related lymphedema: a randomized controlled trial. Breast Cancer Res Treat. 2004; (86): 95-106.

18. Casley-Smith JR. Treatment for lymphedema of the arm: the Casley- Smith method. In: American cancer society lymphedema workshop. N Y Cancer. 1998; (83 Suppl): S2843-60.

19. Segerström K, Bjerle P, Graffman S, Nyström A. Factors that influence the incidence of brachial oedema after treatment of breast cancer. Scand J Plast Reconstr Hand Surg. 1991; (26): 223-27.
20. Camargo MC, Marx AG. Reabilitação física no câncer de mama. São Paulo: Rocca, 2000.

21. Prado MAS. Aderência à atividade física em mulheres submetidas à cirurgia por câncer de mama [Dissertação]. Ribeirão Preto (SP): Universidade de São Paulo. Escola de Enfermagem, 2001.

22. Daley AJ, Mutrie N, Crank H, Coleman R, Saxton J. Exercise therapy in women who have had breast cancer: design of the Sheffield women's exercise and well-being project. Health Educ Res. 2004; 19(6): 686-97.

23. Mckensie DC, Kalda AL. Effect of upper extremity exercise on secondary lymphedema in breast câncer patients: a pilot study. J Clin Oncol. 2003; 21(3): 463-66.

24. Andersen L, Hojris I, Erlandsen M, Andersen J. Treatment of breast-cancer-related lymphedema with or without manual lymphatic drainage: a randomized study. Acta Oncol. 2000; (39): 399-405.

25. Megens A, Harris SR. Physical therapist management of lymphedema following treatment for breast cancer: a critical review of its effectiveness. Phys Ther. 1998; 78(12): 1302-11.

26. Szuba A, Cooke JP, Youssuf SS, Rockson SG. Decongestive lymphatic therapy for patients with cancer-related or primary lymphedema. Am J Med. 2000; (109): 296-300.

27. Kocak Z, Overgaard J. Risk factors of arm lymphedema in breast cancer patients. Acta Oncol. 2000; (39): 389-92.

28. Bunce IH, Mirolo BR, Hennessy JM, Ward LC, Jones LC. Postmastectomy lymphoedema treatment and measurement. Med. J. Aust. 1994; (16): 125-28. 